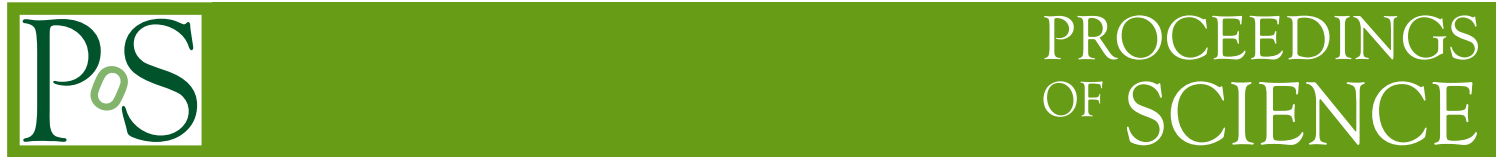

\title{
Minimal doubling and point splitting
}

\author{
Michael Creutz ${ }^{* \dagger}$ \\ Brookhaven Lab \\ E-mail: mike@latticeguy.net
}

Minimally-doubled chiral fermions have the unusual property of a single local field creating two fermionic species. Spreading the field over hypercubes allows construction of combinations that isolate specific modes. Combining these fields into bilinears produces meson fields of specific quantum numbers.

The XXVIII International Symposium on Lattice Filed Theory

June 14-19,2010

Villasimius, Sardinia Italy

*Speaker.

$\dagger$ I am grateful to the Alexander von Humboldt Foundation for support for my ongoing visits to the University of Mainz. This manuscript has been authored under contract number DE-AC02-98CH10886 with the U.S. Department of Energy. Accordingly, the U.S. Government retains a non-exclusive, royalty-free license to publish or reproduce the published form of this contribution, or allow others to do so, for U.S. Government purposes. 


\section{Introduction}

The classic Nielsen-Ninomiya theorem [1] states that for a local lattice action to have an exact chiral symmetry, it must describe an even number of flavors. Within the fundamental Brillouin zone, the fermion propagator should possess an even number of poles. The argument is topological, involving a non-trivial mapping associated with each pole, and this mapping must unwrap elsewhere in the zone. For two space-time dimensions this is a wrapping of a complex number around a circle, while for four dimensions the chiral Dirac operator involves a mapping of quaternions around a sphere $S_{3}$ [2].

Several chiral lattice actions satisfying the minimal condition of $N_{f}=2$ flavors are known. Some time ago Karsten [3] presented a simple form by inserting a factor of $i \gamma_{4}$ into a Wilson like term for space-like hoppings. A slight variation appeared in a discussion by Wilczek [4] a few years later. More recently, I developed a four dimensional action motivated by the analogy with two dimensional graphene. Since then a few more variations were presented by Borici [5], Bedaque et al. [6], and Kimura and Misumi [7]. An effort to classify these various approaches appears in [8].

The main potential advantage of these approaches lies in their ultra-locality. They should be extremely fast in simulations while protecting masses from additive renormalization and helping control mixing of operators with different chirality. The approach also avoids the uncontrolled errors associated with the rooting approximation [9]. On the other hand, all minimally-doubled actions presented so far have the disadvantage of breaking the usual lattice hyper-cubic symmetry. With interactions, this will lead to the necessity of renormalization counter-terms that also violate this symmetry. The extent to which this complicates simulations remains to be investigated.

Here I discuss a point-splitting method for separating the effects of the two flavors which can be created by a single fermion field. For this I will concentrate on the Karsten/Wilczek form as somewhat simpler, but the method should be easily extended to other minimally-doubled formulations.

In the next section I review this action and discuss some of its properties. In section 3 I discuss recent developments on perturbative renormalization and the concomitant counter-terms. Section 4 introduces the proposed point splitting and discusses some of the combinations that create physical particles. Section 5 uses a simple effective Lagrangian argument to discuss some of the lattice artifacts that may occur. Finally section 6 summarizes the basic conclusions.

\section{Karsten/Wilczek fermions}

I concentrate on a minimally-doubled fermion action which is a slight generalization of those presented by Karsten [3] and Wilczek [4]. The fermion term in the lattice action takes the form $\bar{\psi} D \psi$. For free fermions I start with the momentum space form

$$
D(p)=i \sum_{i=1}^{3} \gamma_{i} \sin \left(p_{i}\right)+\frac{i \gamma_{4}}{\sin (\alpha)}\left(\cos (\alpha)+3-\sum_{\mu=1}^{4} \cos \left(p_{\mu}\right)\right)
$$

As a function of the momentum $p_{\mu}$, the propagator $D^{-1}(p)$ has two poles, located at $\vec{p}=0, p_{4}=$ $\pm \alpha$. Relative to the naive fermion action, the other doublers have been given a large "imaginary 
chemical potential." The parameter $\alpha$ allows adjusting the relative positions of the poles. The original Karsten/Wilczek actions correspond to $\alpha=\pi / 2$.

This action maintains one exact chiral symmetry, manifested in the anti-commutation relation $\left[D, \gamma_{5}\right]_{+}=0$. The two species, however, are not equivalent, but have opposite chirality. To see this, expand the propagator around the two poles and observe that one species, that corresponding to $p_{4}=+\alpha$, uses the usual gamma matrices, but the second pole gives a proper Dirac behavior using another set of matrices $\gamma_{\mu}^{\prime}=\Gamma^{-1} \gamma_{\mu} \Gamma$. The Karsten/Wilczek formulation uses $\Gamma=i \gamma_{4} \gamma_{5}$, although other minimally-doubled actions may involve a different transformation. After this transformation $\gamma_{5}^{\prime}=-\gamma_{5}$, showing that the species rotate oppositely under the exact chiral symmetry and thus this symmetry is "flavored." One can think of the physical chiral symmetry as that generated in the continuum theory by $\tau_{3} \gamma_{5}$.

It is straightforward to transform the momentum space action in Eq. (2.1) to position space and insert gauge fields $U_{i j}=U_{j i}^{\dagger}$ on the links connecting lattice sites. Explicitly indicating the site indices, the Dirac operator becomes

$$
D_{i j}=U_{i j} \sum_{\mu=1}^{3} \gamma_{i} \frac{\delta_{i, j+e_{\mu}}-\delta_{i, j-e_{\mu}}}{2}+\frac{i \gamma_{4}}{\sin (\alpha)}\left((\cos (\alpha)+3) \delta_{i j}-U_{i j} \sum_{\mu=1}^{4} \frac{\delta_{i, j+e_{\mu}}+\delta_{i, j-e_{\mu}}}{2}\right)
$$

Note the analogy with spatial Wilson fermions [10] augmented with an $i \gamma_{4}$ insertion in the Wilson term.

\section{Perturbation theory and counter-terms}

Recent perturbative calculations [11] have shown that interactions can shift the relative positions of the poles along the direction between them. In other words, the parameter $\alpha$ receives an additive renormalization. Furthermore, the form of the action treats the fourth direction differently than the spatial coordinates, thus breaking hyper-cubic symmetry along this direction. This suggests three potential new counter-terms for the renormalization of the theory. First there is a possible renormalization of the on-site contribution to the action proportional to $i \bar{\psi} \gamma_{4} \psi$. This provides a handle on the shift of the parameter $\alpha$. Secondly, the breaking of the hyper-cubic symmetry indicates one may need to adjust the fermion "speed of light." This involves a combination of the above on-site term and the strength of temporal hopping proportional to $\delta_{i, j+e_{4}}+\delta_{i, j-e_{4}}$. Finally, the breaking of hyper-cubic symmetry can feed back into the gluonic sector, suggesting a possible counter-term of form $F_{4 \mu} F_{4 \mu}$ to maintain the gluon "speed of light." In lattice language, this corresponds to adjusting the strength of time-like plaquettes relative to space-like ones.

Of these counter-terms, $i \bar{\psi} \gamma_{4} \psi$ is of dimension 3 and is probably the most essential. Quantum corrections induce the dimension 4 terms, suggesting they may be small and could partially be absorbed into accepting a lattice asymmetry. How difficult these counter-terms are to control awaits simulations.

Note that all other dimension 3 counter-terms are forbidden by basic symmetries. For example, chiral symmetry forbids $\bar{\psi} \psi$ and $i \bar{\psi} \gamma_{5} \psi$ terms, and spatial cubic symmetry removes $\bar{\psi} \gamma_{i} \psi, \bar{\psi} \gamma_{i} \gamma_{5} \psi$, and $\bar{\psi} \sigma_{i j} \psi$ terms. Finally, commutation with $\gamma_{4}$ plus space inversion eliminates $\bar{\psi} \gamma_{4} \gamma_{5} \psi$. 


\section{Point splitting}

The fundamental field $\psi$ can create either of the two species. For a quantity that creates only one of them, it is natural to combine fields on nearby sites in such a way as to cancel the other. In other words, one can point split the fields to separate the poles which occur at distinct "bare momenta." For the free theory, one construction that accomplishes this is to consider

$$
\begin{aligned}
& u(q)=\frac{1}{2}\left(1+\frac{\sin \left(q_{4}+\alpha\right)}{\sin (\alpha)}\right) \psi\left(q+\alpha e_{4}\right) \\
& d(q)=\frac{1}{2} \Gamma\left(1-\frac{\sin \left(q_{4}-\alpha\right)}{\sin (\alpha)}\right) \psi\left(q-\alpha e_{4}\right)
\end{aligned}
$$

where $\Gamma=i \gamma_{4} \gamma_{5}$ for the Karsten/Wilczek formulation. Here I have inserted factors containing zeros cancelling the undesired pole. This construction is not unique, and specific details will depend on the particular minimally-doubled action in use. The factor of $\Gamma$ inserted in the $d$ quark field accounts for the fact that the two species use different gamma matrices. This is required since the chiral symmetry is flavored, corresponding to an effective minus sign in $\gamma_{5}$ for one of the species.

It is now straightforward to transform this to position space

$$
\begin{aligned}
& u_{x}=\frac{1}{2} e^{i \alpha x_{4}}\left(\psi_{x}+i \frac{U_{x, x-e_{4}} \psi_{x-e_{4}}-U_{x, x+e_{4}} \psi_{x+e_{4}}}{2 \sin (\alpha)}\right) \\
& d_{x}=\frac{1}{2} \Gamma e^{-i \alpha x_{4}}\left(\psi_{x}-i \frac{U_{x, x-e_{4}} \psi_{x-e_{4}}-U_{x, x+e_{4}} \psi_{x+e_{4}}}{2 \sin (\alpha)}\right)
\end{aligned}
$$

Here I also insert gauge field factors to give simple gauge transformation properties to the pointsplit field. The various phases inserted here serve to remove the oscillations associated with the bare fields having their poles at non-zero momentum.

Given the basic fields for the individual quarks, one can easily construct mesonic fields, which then also involve point splitting. To keep the equations simpler, I now consider the case $\alpha=\pi / 2$. For example, the neutral pion field becomes

$$
\begin{array}{r}
\pi_{0}(x)=\frac{i}{2}\left(\bar{u}_{x} \gamma_{5} u_{x}-\bar{d}_{x} \gamma_{5} d_{x}\right)=\frac{i}{16}\left(4 \bar{\psi}_{x} \gamma_{5} \psi_{x}+\bar{\psi}_{x-e_{4}} \gamma_{5} \psi_{x-e_{4}}+\bar{\psi}_{x+e_{4}} \gamma_{5} \psi_{x+e_{4}}\right. \\
\left.-\bar{\psi}_{x+e_{4}} U U \gamma_{5} \psi_{x-e_{4}}-\bar{\psi}_{x-e_{4}} U U \gamma_{5} \psi_{x+e_{4}}\right) .
\end{array}
$$

Note that this involves combinations of fields at sites separated by either 0 or 2 lattice spacings. In contrast, the $\eta^{\prime}$ takes the form

$$
\begin{aligned}
\eta^{\prime}(x)=\frac{i}{2}\left(\bar{u}_{x} \gamma_{5} u_{x}+\bar{d}_{x} \gamma_{5} d_{x}\right)=\frac{1}{8}( & \bar{\psi}_{x-e_{4}} U \gamma_{5} \psi_{x}-\bar{\psi}_{x} U \gamma_{5} \psi_{x-e_{4}} \\
& \left.-\bar{\psi}_{x+e_{4}} U \gamma_{5} \psi_{x}+\bar{\psi}_{x} U \gamma_{5} \psi_{x+e_{4}}\right)
\end{aligned}
$$


where all terms connect even with odd parity sites. In a recent paper, Tiburzi [12] has discussed how the anomaly, which gives the $\eta^{\prime}$ a mass of order $\Lambda_{q c d}$, can be understood in terms of the necessary point splitting.

\section{Effective Lagrangians and lattice artifacts}

As with Wilson fermions, the minimally-doubled actions have lattice artifacts that can give rise to unusual effects. Adding corresponding terms to effective Lagrangians can give some insight into such $[13,14]$. With Wilson fermions the artifacts can have one of two consequences depending on the sign of a specific term in the effective potential. Either the chiral transition can become first order, or alternatively it can break up into two transitions separated by the parity broken phase predicted by Aoki [15]. It appears that the latter situation is realized with standard Wilson fermions.

Similarly, at first sight there are two possibilities here as well. These artifacts break isospin symmetry, allowing the charged pion mass to differ from that of the neutral pion. The simplest situation has the charged pions heavier. In this case as the quark mass goes to zero the neutral pion becomes a conventional Goldstone boson associated with the one exact chiral symmetry of the theory. Alternatively, if the charged pions are lighter, then before one reaches the chiral limit their mass can go to zero and the charged mesons would condense. As with the Aoki phase, there would then be a region of spontaneous parity breaking.

One qualitative difference between Wilson fermions and the minimally-doubled case is that in the latter situation the fermion determinant is strictly positive. While it does not directly apply because Lorentz invariance has been broken by the lattice artifacts, the Vafa/Witten argument [16] suggests that the first alternative is the more likely. Lattice simulations should be able to settle which situation is the case.

\section{Summary}

Minimally-doubled fermion actions present the possibility of fast simulations while maintaining one exact chiral symmetry. They do, however, introduce some peculiar aspects. An explicit breaking of hyper-cubic symmetry allows additional counter-terms to appear in the renormalization. While a single field creates two different species, spreading this field over nearby sites allows isolation of specific states and the construction of physical meson operators. Finally, lattice artifacts break isospin and give two of the three pseudoscalar mesons an additional contribution to their mass. Depending on the sign of this mass splitting, one can either have a traditional Goldstone pseudoscalar meson or a parity breaking Aoki-like phase.

\section{References}

[1] H. B. Nielsen and M. Ninomiya, Absence Of Neutrinos On A Lattice. 1. Proof By Homotopy Theory, Nucl. Phys. B 185, 20 (1981) [Erratum-ibid. B 195, 541 (1982)].

[2] M. Creutz, Four-dimensional graphene and chiral fermions, JHEP 0804, 017 (2008) [arXiv:0712.1201 [hep-lat]].

M. Creutz, Local chiral fermions, PoS LATTICE2008, 080 (2008) [arXiv:0808.0014 [hep-lat]]. 
[3] L. H. Karsten, Lattice Fermions In Euclidean Space-Time, Phys. Lett. B 104, 315 (1981).

[4] F. Wilczek, On Lattice Fermions, Phys. Rev. Lett. 59, 2397 (1987).

[5] A. Borici, Creutz Fermions On An Orthogonal Lattice, Phys. Rev. D 78, 074504 (2008) [arXiv:0712.4401 [hep-lat]].

[6] P. F. Bedaque, M. I. Buchoff, B. C. Tiburzi and A. Walker-Loud, Phys. Rev. D 78, 017502 (2008) [arXiv:0804.1145 [hep-lat]].

[7] T. Kimura and T. Misumi, Lattice Fermions Based on Higher-Dimensional Hyperdiamond Lattices, Prog. Theor. Phys. 123, 63 (2010) [arXiv:0907.3774 [hep-lat]].

T. Kimura and T. Misumi, Characters of Lattice Fermions Based on the Hyperdiamond Lattice, arXiv:0907.1371 [hep-lat]. See also the presentation by Misumi at this conference.

[8] M. Creutz and T. Misumi, Classification of Minimally Doubled Fermions, arXiv:1007.3328 [hep-lat].

[9] M. Creutz, Chiral anomalies and rooted staggered fermions, Phys. Lett. B 649 (2007) 230 [arXiv:hep-lat/0701018].

M. Creutz, Why rooting fails, PoS LATTICE2007, 007 (2006) [arXiv:0708.1295 [hep-lat]].

[10] K. G. Wilson, Quarks And Strings On A Lattice, in New Phenomena In Subnuclear Physics. Part A. Proceedings of the First Half of the 1975 International School of Subnuclear Physics, Erice, Sicily, July 11 - August 1, 1975, ed. A. Zichichi, Plenum Press, New York, 1977, p. 69.

[11] S. Capitani, M. Creutz, J. Weber and H. Wittig, Renormalization of minimally doubled fermions, arXiv:1006.2009 [hep-lat]. See also the presentations by Capitani and Weber at this conference.

[12] B. C. Tiburzi, Chiral Lattice Fermions, Minimal Doubling, and the Axial Anomaly, arXiv:1006.0172 [hep-lat].

[13] M. Creutz, Wilson fermions at finite temperature, arXiv:hep-lat/9608024.

[14] S. R. Sharpe and R. L. . Singleton, Spontaneous flavor and parity breaking with Wilson fermions, Phys. Rev. D 58, 074501 (1998) [arXiv:hep-lat/9804028].

[15] S. Aoki, New Phase Structure For Lattice QCD With Wilson Fermions, Phys. Rev. D 30, 2653 (1984).

[16] C. Vafa and E. Witten, Parity Conservation In QCD, Phys. Rev. Lett. 53, 535 (1984). 